Department of Applied Physics, Twente University of Technology, Enschede (The Netherlands)

\title{
Calculation of the effect of inertia on the dynamic viscosity of dilute emulsions in a pure straining motion
}

\author{
M. Oosterbroek, R. Tropper, and J. Mellema
}

With 5 figures

(Received July 17, 1979)

\begin{tabular}{|c|c|}
\hline Notation & \\
\hline$a$ & radius of the emulsion droplets \\
\hline$b$ & radius of the unit cell \\
\hline$h_{i}^{(+)}$ & spherical Hankel function of the first kind \\
\hline$i$ & $=\sqrt{-1}$ \\
\hline$j_{l}$ & spherical Bessel function \\
\hline$k$ & $\begin{array}{l}\text { complex wave number in the region } \\
a<r<b\end{array}$ \\
\hline$m$ & mass \\
\hline$n$ & local unit normal vector \\
\hline$p_{0}$ & hydrostatic pressure in the region $a<r<b$ \\
\hline$p$ & pressure in the region $a<r<b$ \\
\hline$q$ & real constant \\
\hline$r$ & radial coordinate in spherical coordinates \\
\hline$r$ & position vector \\
\hline$t$ & time \\
\hline$u$ & $\begin{array}{l}\text { physical component of the radial velocity in } \\
\text { the region } a<r<b\end{array}$ \\
\hline$v$ & $\begin{array}{l}\text { physical component of the tangential veloc- } \\
\text { ity in the region } a<r<b\end{array}$ \\
\hline $\boldsymbol{v}$ & velocity vector \\
\hline$x$ & element of $a \mathbb{C}_{8}$ space \\
\hline$A, B, C, D$ & $\begin{array}{l}\text { time-dependent coefficient of the flow field in } \\
\text { the region } a<r<b\end{array}$ \\
\hline$A_{h}^{*}$ & time-dependent coefficient \\
\hline$D_{0}^{*}$ & $\begin{array}{l}\text { time-independent part of a rate of strain } \\
\text { tensor }\end{array}$ \\
\hline$D$ & rate of strain tensor \\
\hline $\boldsymbol{M}, \widetilde{\boldsymbol{M}}$ & $8 \times 8$ matrix \\
\hline$O$ & $6 \times 6$ matrix \\
\hline$O$ & surface \\
\hline$P$ & $6 \times 2$ matrix \\
\hline$P$ & rate of work \\
\hline $\begin{array}{l}P_{2} \\
P_{2}^{\prime}\end{array}$ & $\begin{array}{l}\text { Legendre polynomial of the second order } \\
=d P_{2} / d \theta\end{array}$ \\
\hline$Q$ & $2 \times 6$ matrix \\
\hline $\bar{R}$ & $2 \times 2$ matrix \\
\hline$R$ & radius of volume $V$ \\
\hline Re & Reynolds number \\
\hline$T$ & real time-independent constant \\
\hline$\hat{T}$ & stress tensor in the region $a<r<b$ \\
\hline$\tilde{T}$ & $\begin{array}{l}\text { deviatoric stress tensor in the region } \\
a<r<b\end{array}$ \\
\hline $\begin{array}{l}\hat{T}\langle r r\rangle, \hat{T} \\
\hat{T}\langle\theta \theta\rangle, \hat{T}\end{array}$ & $\begin{array}{ll}\langle r \theta\rangle, \hat{T}\langle r \phi\rangle & \text { stress tensor components in } \\
\langle\theta \phi\rangle, \hat{T}\langle\phi \phi\rangle & \text { spherical coordinates in the } \\
& \text { region } a<r<b\end{array}$ \\
\hline
\end{tabular}

$T\langle r r\rangle, T\langle r \theta\rangle, T\langle r \phi\rangle \quad$ deviatoric stress tensor com-

$T\langle\theta \theta\rangle, T\langle\theta \phi\rangle, T\langle\phi \phi\rangle$ ponents in spherical coordi-

$\alpha \quad$ angle

$\gamma \quad$ interfacial tension

$\eta \quad$ Newtonian viscosity in the region $a<r<b$

$\eta^{*} \quad$ dynamic viscosity of the emulsion

$\eta_{d}^{*} \quad$ dimensionless dynamic viscosity of the emulsion

$\eta_{01}^{*}$ dimensionless dynamic viscosity of the emulsion without inertial effects

$\eta_{0}^{*} \quad=\eta_{0}^{\prime}-i \eta_{0}^{\prime \prime}$, dynamic viscosity of a massdashpot system (fig. 5)

$\theta \quad$ angle measured downwards from the $z$ axis

(spherical coordinates)

$\lambda$ damping constant of a dashpot

$\lambda_{d} \quad$ dimensionless time constant

$\rho$ density in the region $a<r<b$

$\rho_{d} \quad$ dimensionless density of the emulsion

$\Phi \quad$ volume fraction

$\phi \quad$ angle measured around the $z$-axis (spherical coordinates)

$\omega$. angular frequency

$\omega_{d}$ dimensionaless angular frequency

Superscripts
* quantity pertinent to region $r<a$
* quantity pertinent to region $r>b$ or to the homogeneous volume (fig. 1, I)

\section{Introduction}

Viscoelastic behaviour of dilute emulsions has been predicted by several authors $(1-4)$. In their theories they have used the Stokes equation to solve the hydrodynamic problem, thus neglecting influence of inertia.

Consider a harmonically oscillating flow in the neighbourhood of a droplet with radius $a$. The flow field is characterized by $a$ rate of strain tensor $\boldsymbol{D}$. The ratio between the linear 
inertia term and the viscous term in the NavierStokes equation [1] is on the order of $\omega a^{2} \rho / \eta$,

$\rho\left\{\frac{\partial v}{\partial t}+v \cdot \nabla v\right\}=-\nabla p+\eta \Delta v$.

The ratio between the non-linear inertia term and the viscous term in eq. [1] is on the order of $|\boldsymbol{D}| a^{2} \rho / \eta$. These ratios can be varied independently with the aid of $\omega$ en $|\boldsymbol{D}|$. For sufficiently small rates of strain the non-linear inertia term can be neglected, whereas the linear inertia term can be important.

In this paper the influence of the linear inertia term on the dynamic viscosity $\eta^{*}$ of dilute emulsions in an oscillating pure straining motion will be investigated.

In our calculation of $\eta^{*}$ we used the cell model of Fröhlich and Sack (5).

\section{Definition of the problem}

\subsection{The cell model (5)}

In the application of the cell model of Fröhlich and Sack (5) to our calculation of the mechanical properties of an emulsion two spherical volumes, radius $R$, are compared. The liquid of one of these volumes (I, in fig. 1) is conceived as homogeneous. It possesses the macroscopic mechanical properties of the emulsion. The other sphere (II, in fig. 1) contains three liquids. In the centre a droplet, radius $a$, is present. It is surrounded by a concentric layer of the external phase. The outer radius of the external phase is taken as $b=\Phi^{-1 / 3} a$. This configuration of the internal and external phases makes up the unit cell of the cell model. The cell is surrounded by the same homogeneous liquid which fills volume I.

A harmonically oscillating pure straining motion is imposed on the liquid in volume I.
The general solution for a harmonically oscillating flow is well-known (6) and can be given for the three liquids in volume II.

Typical for the cell model considered is that only that part of the general solution in volume II for $r>b$ is allowed which gives that the difference between the velocity (respectively stress) components on $r=R$ of the volumes I and II divided by the similar components of the homogeneous volume I equals $o\left(R^{-3}\right)$. The difference reflects the influence of the cell on the flow field for $r>b$ in II compared with the undisturbed field of I. The flow field II, restricted by the mentioned boundary conditions at $r=R$, distorts the spheres at $r=a$ and $r=b$. The distortion is taken very small.

Then from the boundary conditions at the distorted spheres at $r=a$ and $r=b$ the mechanical properties of the emulsion can be calculated. They can be expressed in $\omega, \rho, \eta$, $\eta^{\prime}, \gamma, \alpha$, and $\Phi$.

\subsection{Starting points and assumptions}

Oldroyd (1) applied the cell model of Fröhlich and Sack (5) to the calculation of the mechanical properties of a dilute emulsion without inertia effects. In the past years the properties derived appeared, both experimentally and theoretically, to be valid in the first order of concentration. Since in this paper we use the same method, our results will also be valid only in the first order of concentration. Hence the results are only relevant for sufficiently diluted emulsions in which hydrodynamic interaction between particles is negligible.

The emulsion consists of spherical drops of a Newtonian fluid immersed in a second Newtonian fluid. Both fluids are incompressible:

$\operatorname{div} v=\operatorname{div} \boldsymbol{v}^{\prime}=0$.
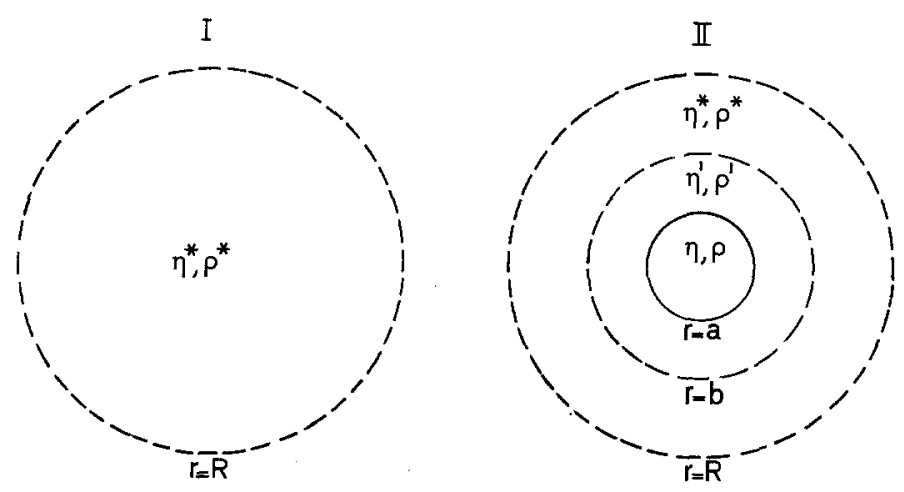

Fig. 1. The volumes for the homogeneous (I) and composite (II) fluid 
Densities of internal and external phases are taken to be the same $\left(\rho=\rho^{\prime}=\rho^{*}\right)$ in order to avoid translational motion of the centres of mass of the emulsion droplets relative to the external phase.

We restrict ourselves to oscillating pure straining motion. More specifically at $r \geq R$ we impose a harmonically oscillating velocity field $v$ on the emulsion of volume I of the form

$\boldsymbol{v}^{*}(\boldsymbol{r}, t)=D_{0}^{*} \cdot \boldsymbol{r} \exp i \omega t$

$D_{0}^{*}$ is a time- and position-independent real symmetric traceless tensor of the second order and $r$ is the position vector with respect to the centre of volume I. Complex notation is used throughout the calculations.

The order of magnitude of the velocity components in volume II can be estimated with the aid of eq. [3] because the velocity at $r=R$ in volume II approaches [3] for $r=R \rightarrow \infty$. The magnitude of $\boldsymbol{D}_{0}^{*}$ is limited in our problem due to several assumptions. Velocities and pressures inside $(r<a)$ and outside $(a<r<b)$ the droplet are calculated with the Navier-Stokes equation $[1]$, in which the non-linear inertia term is neglected. This is justified if $\left|D_{0}^{*}\right| a^{2} \rho / \eta \ll 1$. In a harmonically oscillating flow field the NavierStokes equation then reduces to

$i \omega \rho \boldsymbol{v}=-\nabla p+\eta \Delta v$.

The droplets in the emulsion are deformed by the flow. We confine the calculations to almost spherical droplets. It is easy to demonstrate that one can find sufficiently small values of $\left|D_{0}^{*}\right|$ to make the assumption realistic.

Further we assume that for every value of $\omega$ under consideration the magnitude of $D_{0}^{*}$ is so small that the macroscopically observed mechanical response of the emulsion in the velocity field [3] can be described by the dynamic viscosity $\eta^{*}$. Hence the results of the calculations are only valid for $\left|D_{0}^{*}\right|$ values smaller than the most stringent limit determined by the assumptions previously mentioned. For the homogeneous fluid the relation between the stress tensor $\hat{T}^{*}$ and the rate of strain tensor $D^{*}$ is

$\hat{\boldsymbol{T}}^{*}=-p \mathbf{1}+2 \eta^{*} \boldsymbol{D}^{*}$

and the deviatoric part of $\hat{T}^{*}$ is

$T^{*}=2 \eta^{*} D^{*}$.

Since [5] is a linear relationship, eq. [4] also holds for the homogeneous fluid if $\rho, v, p$, and $\eta$ are replaced by $\rho^{*}, \boldsymbol{v}^{*}, p^{*}$, and $\eta^{*}$. The stress tensor compatible to the velocity field [3] can be calculated with the aid of the relations [4] (modified) and [5]:

$\hat{\boldsymbol{T}}^{*}=\left\{-\frac{1}{2} i \omega \rho\left(\boldsymbol{r} \cdot \boldsymbol{D}_{0}^{*} \cdot \boldsymbol{r}\right) \mathbf{1}+2 \eta^{*} \boldsymbol{D}_{0}^{*}\right\} \exp i \omega t$.

The deviatoric part of $\hat{\boldsymbol{T}}^{*}$ can be written as the sum of six independent tensors which can be transformed into each other by rotation and reflection of the frame of reference. The linearity of the relations [3], [4], and [5] makes it possible to solve our problem by imposing one of the special stress tensors given in ref. (1) on the emulsion instead of the linear velocity field [3]. We impose (in Cartesian coordinates) the tensor field

$\left(\boldsymbol{T}^{*}\right)=\left(\begin{array}{rrr}2 T & 0 & 0 \\ 0 & -T & 0 \\ 0 & -0 & -T\end{array}\right) \exp i \omega t$,

in which $T$ is a real constant.

This tensor imposed on the fluid is only compatible with an axisymmetrical flow field both for the homogeneous and for the composite element (see fig.1). The spherical form of the droplets promotes the use of spherical coordinates, which will be used in the next sections.

\section{The stress and velocity field}

\subsection{Homogeneous volume (fig. 1, I)}

In spherical coordinates the time-independent deviatoric part of the imposed stress field [8] is $\left.\begin{array}{l}T^{*}\langle r r\rangle=2 T P_{2}(\cos \theta), \\ T^{*}\langle\theta \theta\rangle=T\left(1-2 P_{2}(\cos \theta)\right), \\ T^{*}\langle\phi \phi\rangle=-T, \\ T^{*}\langle r \theta\rangle=T P_{2}^{\prime}(\cos \theta), \\ T^{*}\langle r \phi\rangle=T^{*}\langle\theta \phi\rangle=0 .\end{array}\right\}$

The radial velocity $u^{*}$, the tangential velocity $v^{*}$, and the pressure $p^{*}$, derived from eqs. [3], [6], and [4] and being in agreement with [9], are

$$
\begin{aligned}
& u^{*}=T P_{2}(\cos \theta) r / \eta^{*}, \\
& v^{*}=\frac{1}{2} T P_{2}^{\prime}(\cos \theta) r / \eta^{*}, \\
& p^{*}=\frac{1}{2} T k^{* 2} r^{2} P_{2}(\cos \theta)+p_{0}^{*} \\
& \text { where } k^{* 2}=-i \omega \rho / \eta^{*} .
\end{aligned}
$$


In the next sections the angular dependence of the fields in [9] and [10] will be indicated as $P_{2}$-axial symmetry.

\subsection{Composite volume (fig. 1, II)}

The general solution of the velocity and stress field for the $P_{2}$-axial symmetry of [9] and [10] is given by $\operatorname{Lamb}(6)$. The formulae for $a<r<b$ are shown in spherical coordinates in Appendix 1. Similar formulae apply in the regions $0<r<a$ and $b<r<R$ with constants having primes and asterisks respectively. Hence the general solution for the composite volume contains 15 time-dependent unknowns: $A^{*}, A^{\prime}, A, B^{*}, B^{\prime}, B$, $C^{*}, C^{\prime}, C, D^{*}, D^{\prime}, D, P_{0}^{*}, p_{0}^{\prime}, p_{0}$, and further the unknown $\eta^{*}$.

\subsection{Boundary and cell model conditions for the composite volume}

There are boundary conditions which apply at the interface droplet-external phase and at the interface external phase-homogeneous fluid. At both interfaces we require continuity of tangential and normal velocity components. The requirement of force equilibrium at an interface element gives conditions for tangential and normal stress components. The tangential stress should be continuous at both interfaces. At the interface droplet-external phase the interfacial tension $\gamma$ causes a discontinuity in the normal stress component, which depends on the curvature of the drop surface. The Laplace equation gives the relationship between the hydrostatic pressures $p_{0}$ and $p_{0}^{\prime}$ in the usual way:

$p_{0}=p_{0}^{\prime}-2 \gamma / a$.

At the artificial cell boundary on the other hand we require continuity of the normal stress component.

The above-mentioned conditions result in 9 equations. Inside the drop the velocity should be finite at $r=0$. It follows that $B^{\prime}=C^{\prime}=0$. At $r=R$ the conditions typical for the cell model are applicable. According to Fröhlich and Sack (5) the difference between the corresponding velocity respectively stress components of the homogeneous and composite volume at $r=R$ divided by the similar components of the homogeneous volume at $r=R$ should be equal to $o\left(R^{-3}\right)$. In contrast with the problem in which the density equals zero, here two possible sets of the remaining unknowns instead of one are left:

1. $A^{*}=B^{*}=0, D^{*}=T / \eta^{*}, p_{0}^{*}=0$.

2. $A^{*}=i B^{*}, \quad C^{*}=0, \quad D^{*}=T / \eta^{*}$, $p_{0}^{*}=0$.

We can distinguish between these possibilities by considering the rate of work

$P=\int_{\partial \boldsymbol{V}} \boldsymbol{v} \cdot T \cdot \boldsymbol{n} d 0$

done at $r=R$ on the homogeneous and composite element.

We require that the difference between the rates of work done on the homogeneous and the composite volume at $r=R$ vanishes for $R \rightarrow \infty$, which was also the case, but not explicitly required, for Oldroyd's solution without inertia. This cancels the first possibility.

Before we can give the equations for the boundary conditions we have to calculate the discontinuity in the normal stress at the interface droplet-internal phase. The shape of the drop surface is given by

$$
\begin{aligned}
r= & a+\left\{A^{\prime} a^{-1} j_{2}\left(k^{\prime} a\right) / i \omega+D^{\prime} a / i \omega\right\} \\
& \cdot P_{2}(\cos \alpha), \\
\theta= & \alpha+\frac{1}{6}\left\{A^{\prime}\left(3 a^{-1} j_{2}\left(k^{\prime} a\right)-k^{\prime} j_{3}\left(k^{\prime} a\right)\right) / i \omega\right. \\
& \left.+3 D^{\prime} a / i \omega\right\} P_{2}^{\prime}(\cos \alpha) .
\end{aligned}
$$

Then the pressure difference - the static pressure being omitted - at $r=a$ becomes:

$$
\begin{aligned}
p^{\prime}-p= & 2 \gamma a^{-1}\left\{1+2\left(A^{\prime} a^{-2} j_{2}\left(k^{\prime} a\right)+D^{\prime}\right)\right. \\
& \left.\cdot P_{2}(\cos \theta) / i \omega\right\} .
\end{aligned}
$$

The remaining eight boundary conditions for the velocity and stress components are, at $r=a$ :

$$
\begin{aligned}
& \hat{T}\langle r r\rangle: \eta^{\prime}\left\{2 A^{\prime}\left(a^{-2} j_{2}\left(k^{\prime} a\right)-k^{\prime} a^{-1} j_{3}\left(k^{\prime} a\right)\right)\right. \\
& \left.+D^{\prime}\left(2-\frac{k^{\prime 2} a^{2}}{2}\right)\right\} \\
& +4 \gamma(i \omega a)^{-1}\left(A^{\prime} a^{-2} j_{2}\left(k^{\prime} a\right)+D^{\prime}\right) \\
& =\eta\left\{2 A\left(a^{-2} j_{2}(k a)-k a^{-1} j_{3}(k a)\right)\right. \\
& +2 B\left(a^{-2} j_{-3}(k a)+k a^{-1} j_{-4}(k a)\right) \\
& +C\left(-8 a^{-5}+\frac{k^{2} a^{-3}}{3}\right) \\
& \left.+D\left(2-\frac{k^{2} a^{2}}{2}\right)\right\}
\end{aligned}
$$




$$
\begin{aligned}
& \hat{T}\langle r \theta\rangle: \eta^{\prime}\left\{A^{\prime}\right.\left(\left(a^{-2}-\frac{k^{\prime 2}}{6}\right) j_{2}\left(k^{\prime} a\right)\right. \\
&\left.\left.+\frac{a^{-1} k^{\prime}}{3} j_{3}\left(k^{\prime} a\right)\right)+D^{\prime}\right\} \\
&=\left\{A \left(\left(a^{-2}-\frac{k^{2}}{6}\right) j_{2}(k a)\right.\right. \\
&\left.+\frac{a^{-1} k}{3} j_{3}(k a)\right) \\
&+B\left(\left(a^{-2}-\frac{k^{2}}{6}\right) j_{-3}(k a)\right. \\
&\left.-\frac{a^{-1} k}{3} j_{-4}(k a)\right) \\
&\left.+\frac{8}{3} C a^{-5}+D\right\} ; \\
& A^{\prime} a^{-1} j_{2}\left(k^{\prime} a\right)+D^{\prime} a \\
&= A a^{-1} j_{2}(k a)+B a^{-1} j_{-3}(k a) \\
&+C a^{-4}+D a ; \\
& A^{\prime}\left(3 a^{-1} j_{2}\left(k^{\prime} a\right)-k^{\prime} j_{3}\left(k^{\prime} a\right)\right)+3 D^{\prime} a \\
&= A\left(3 a^{-1} j_{2}(k a)-k j_{3}(k a)\right) \\
&+B\left(3 a^{-1} j_{-3}(k a)+k j_{-4}(k a)\right) \\
&-2 C a^{-4}+3 D a ; \\
& v: \quad
\end{aligned}
$$

and at $r=b$ :

$$
\begin{aligned}
& \hat{T}\langle r r\rangle: \eta A\left\{2\left(b^{-2} j_{2}(k b)-k b^{-1} j_{3}(k b)\right)\right. \\
& +2 B\left(b^{-2} j_{-3}(k b)+k b^{-1} j_{-4}(k b)\right) \\
& +C\left(8 b^{-5}+\frac{k^{2} b^{-3}}{3}\right) \\
& \left.+D\left(2-\frac{k^{2} b^{2}}{2}\right)\right\} \\
& =\eta^{*} A_{h}^{*}\left\{\left(2 b^{-2} h_{2}^{+}\left(k^{*} b\right)\right.\right. \\
& \left.-2 k^{*} b^{-1} h_{3}^{+}\left(k^{*} b\right)\right) \\
& \left.+D^{*}\left(2-\frac{k^{* 2} b^{2}}{2}\right)\right\} \\
& \hat{T}\langle r \theta\rangle: \eta\left\{A \left(\left(b^{-2}-\frac{k^{2}}{6}\right) j_{2}(k b)\right.\right. \\
& \left.+\frac{b^{-1} k}{3} j_{3}(k b)\right) \\
& +B\left(\left(b^{-2}-\frac{k^{2}}{6}\right) j_{-3}(k b)\right.
\end{aligned}
$$

$$
\begin{aligned}
& \left.-\frac{b^{-1} k}{3} j_{-4}(k b)\right) \\
& \left.+\frac{8}{3} C b^{-5}+D\right\} \\
= & \eta^{*}\left(A _ { h } ^ { * } \left(\left(b^{-2}-\frac{k^{* 2}}{6}\right) h_{2}^{+}\left(k^{*} b\right)\right.\right. \\
& \left.\left.+\frac{b^{-1} k^{*}}{3} h_{3}^{+}\left(k^{*} b\right)\right)+D^{*}\right\} ;[20] \\
A b^{-1} & j_{2}(k b)+B b^{-1} j_{-3}(k b) \\
& +C b^{-4}+D b \\
= & A_{h}^{*} b^{-1} h_{2}^{+}\left(k^{*} b\right)+D^{*} b ; \\
A\left(3 b^{-1} j_{2}(k b)-k j_{3}(k b)\right) & \\
& +B\left(3 b^{-1} j_{-3}(k b)+k j_{-4}(k b)\right) \\
& -2 C b^{-4}+3 D b \\
= & A_{h}^{*}\left(3 b^{-1} h_{2}^{+}\left(k^{*} b\right)-k^{*} h_{3}^{+}\left(k^{*} b\right)\right) \\
& +3 D^{*} b ;
\end{aligned}
$$

$h_{2}^{+}$and $h_{3}^{+}$represent spherical Hankel functions defined by

$h_{2}^{+}(z)=j_{-3}(z)+i j_{2}(z)$,

$h_{3}^{+}(z)=-j_{-4}(z)+i j_{3}(z)$.

\section{Determination of $\eta^{*}$}

An implicit expression for $\eta^{*}$ can be found by applying a condition so that the set of eight boundary conditions [15]-[22] yields a nonzero solution for the unknowns

$A^{\prime}, D^{\prime}, A, B, C, D, A_{h}^{*}$, and $D^{*}$.

This conditions is that the determinant of the coefficients of the unknowns of the set equals zero. The derived implicit expression for $\eta^{*}$ has no analytical solution. Therefore we have computed $\eta^{*}$ numerically. It is convenient to use dimensionless quantities in the numerical calculation. We made all quantities dimensionless with $a_{3} \eta$, and $\gamma$. The dimensionless character of a quantity is indicated by an index $d$. Some important quantities are: $\omega_{d}=\omega a \eta / \gamma, \rho_{d}=$ $\rho a \gamma / \eta^{2}, \eta_{d}^{\prime}=\eta^{\prime} / \eta, \eta_{d}^{*}=\eta^{*} / \eta$.

Let us now reformulate our problem. If the unknowns of [23] are represented by an element $x \in \mathbb{C}_{8}$ and the coefficients of the unknowns of the set of eqs. [15] $-[22]$ by an $8 \times 8$ matrix $\boldsymbol{M}$, the boundary conditions in 3.3 can be given as $\boldsymbol{M} \cdot \boldsymbol{x}=0$. 
The implicit expression for $\eta_{d}^{*}$ is given by $\operatorname{det} \boldsymbol{M}=0$. We transform $\boldsymbol{M}$ with row and column manipulation into $\tilde{M}$ in such a way that for $\rho_{d} \rightarrow 0$ the coefficient matrix of Oldroyd's boundary conditions (1) is obtained and $\tilde{M}_{77}$, $\tilde{M}_{78}, \tilde{M}_{87}$, and $\tilde{M}_{88}$ are the only $\eta_{d}^{*}$-dependent elements within this limit. Now we split $\tilde{M}$ into four submatrices $\boldsymbol{O}(6 \times 6), \boldsymbol{P}(6 \times 2), Q(2 \times 6)$, and $\boldsymbol{R}(2 \times 2)$. The matrix $\boldsymbol{R}$ is given by $\tilde{M}_{77}$, $\tilde{M}_{78}, \tilde{M}_{87}$, and $\tilde{M}_{88} ;$ the other ones are defined by

$(\tilde{M})=\left(\begin{array}{c:c}\boldsymbol{O} & \boldsymbol{P} \\ \hdashline \boldsymbol{Q} & \boldsymbol{R}\end{array}\right)$.

Linear algebraic operations give:

$\operatorname{det}\left(\boldsymbol{Q} \boldsymbol{O}^{-1} \boldsymbol{P}+\boldsymbol{R}\right)=0$.

The determinant in [26] is that of a $2 \times 2$ matrix. It gives a simple quadratic expression in $\eta_{d}^{*}$ for Oldroyd's case.

If $\rho_{d} \neq 0$, spherical Hankel functions depending on $\eta_{d}^{*}$ enter the elements of $\boldsymbol{P}$ and $\boldsymbol{R}$ as additional terms. Now an iterative procedure is used for obtaining $\eta_{d}^{*}$.

A start value of $\eta_{d}^{*}$ is put into the Hankel functions. The quadratic expression in $\eta_{d}^{*}$ (eq. [26]) is solved. The value of $\eta_{d}^{*}$ thus obtained is now put into the Hankel functions. Again the quadratic expression in $\eta_{d}^{*}$ is solved and a better value of $\eta_{d}^{*}$ than the previous ones is obtained, etc. This iteration converges very quickly. For low frequencies we use Oldroyd's values of $\eta_{d}^{*}$ as start values. For higher frequencies we use the $\eta_{d}^{*}$ from a calculation at a lower frequency as a start value. However, the iteration result is not even dependent on large changes in the start value.

For large arguments of the Bessel and Hankel functions the numerical results were not consistent compared with results for smaller values of the arguments. This problem is related to the limited computing accuracy, which could be demonstrated by comparison of results obtained from computers using a different precision (DEC10 and CDC computer). Unfortunately this problem limits the ranges of parameter values for which calculation is possible.

\section{Discussion of the results}

In figure 2 the linearity of $\eta_{d}^{*}$ as a function of $\Phi$ is shown for several values of the parameters $\rho_{d}, \omega_{d}$, and $\eta_{d}^{\prime}$. No significant deviations

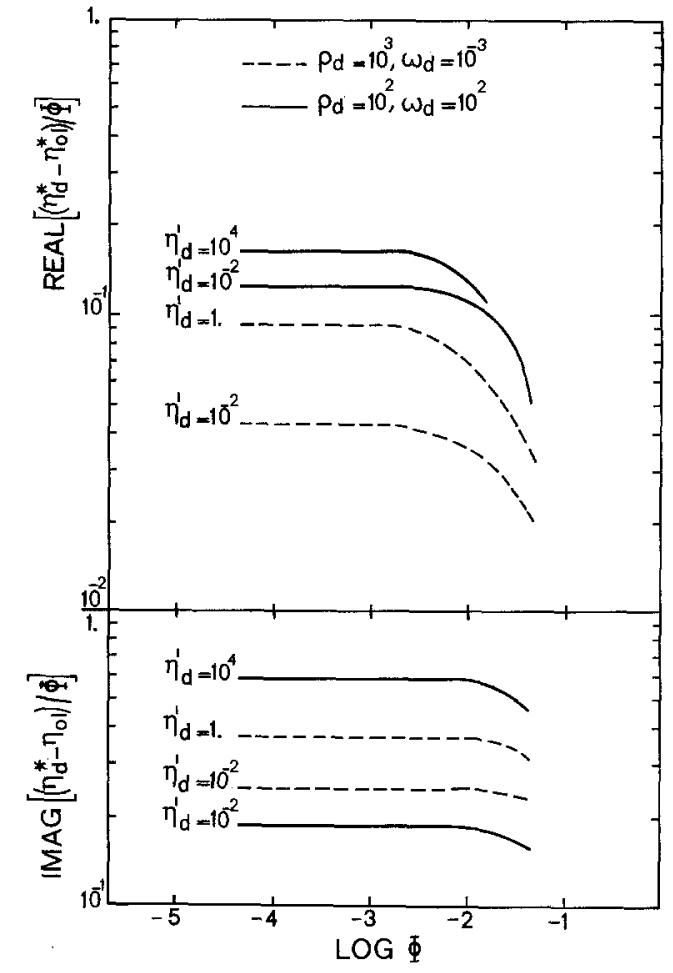

Fig. 2. The calculated correction $\left(\eta_{d}^{*}=\eta_{01}\right) / \Phi$ as a function of $\Phi$ for various values of the parameters $\rho_{d}, \omega_{d}$, and $\eta_{d}^{\prime}$

from linearity were found for concentrations up to about $5 \cdot 10^{-3}$. For this $\Phi$ range the dependence of $\left(\eta_{d}^{*}-1\right) / \Phi$ of $\omega_{d}$ is shown for several $\eta_{d}^{\prime}$ and $\rho_{d}$ values in figure 3 and figure 4 . We verified that for small values of $\omega_{d}$ or $\rho_{d}$ our results approach the solution of the problem without inertia forces as given by oldroyd (1) $\left(\omega_{d} \rho_{d} \ll 1\right.$ in the Bessel and Hankel functions):

$$
\begin{aligned}
& \frac{\eta_{01}^{*}-1}{\Phi} \\
& =\frac{5\left(4\left(2+5 \eta_{d}^{\prime}\right)-i\left(1-\eta_{d}^{\prime}\right)\left(16+19 \eta_{d}^{\prime}\right) \omega_{d}\right)}{40\left(1+\eta_{d}^{\prime}\right)+i\left(3+2 \eta_{d}^{\prime}\right)\left(16+19 \eta_{d}^{\prime}\right) \omega_{d}}
\end{aligned}
$$

The viscoelastic behaviour of eq. [27] of a dilute emulsion (without inertia forces) shows appreciable changes in $\left(\eta_{d}^{*}-1\right) / \Phi$ for $\omega_{d}$ values which are approximately equal to the inverse of the characteristic time

$\lambda_{d}=\frac{\left(16+19 \eta_{d}^{\prime}\right)\left(3+2 \eta_{d}^{\prime}\right)}{40\left(1+\eta_{d}^{\prime}\right)}$.

These phenomena occur when the interfacial tension forces are on the same order of magni- 


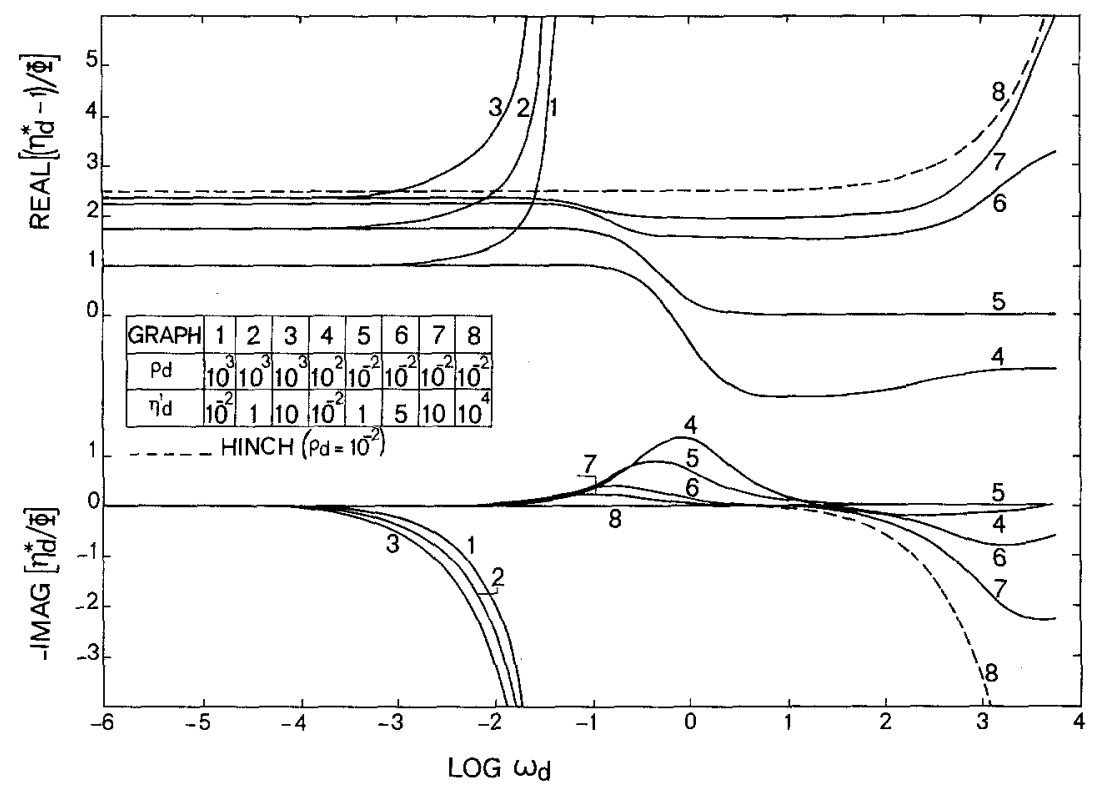

Fig. 3. The real and imaginary parts of $\left(\eta_{d}^{*}-1\right) / \Phi$ as a function of $\omega_{d}$ for various values of the parameter $\rho_{d}$ and $\eta_{d}^{\prime}$. The dashed line coincides with the analytical solution given by Hinch for $\rho_{d}=10^{-2}$

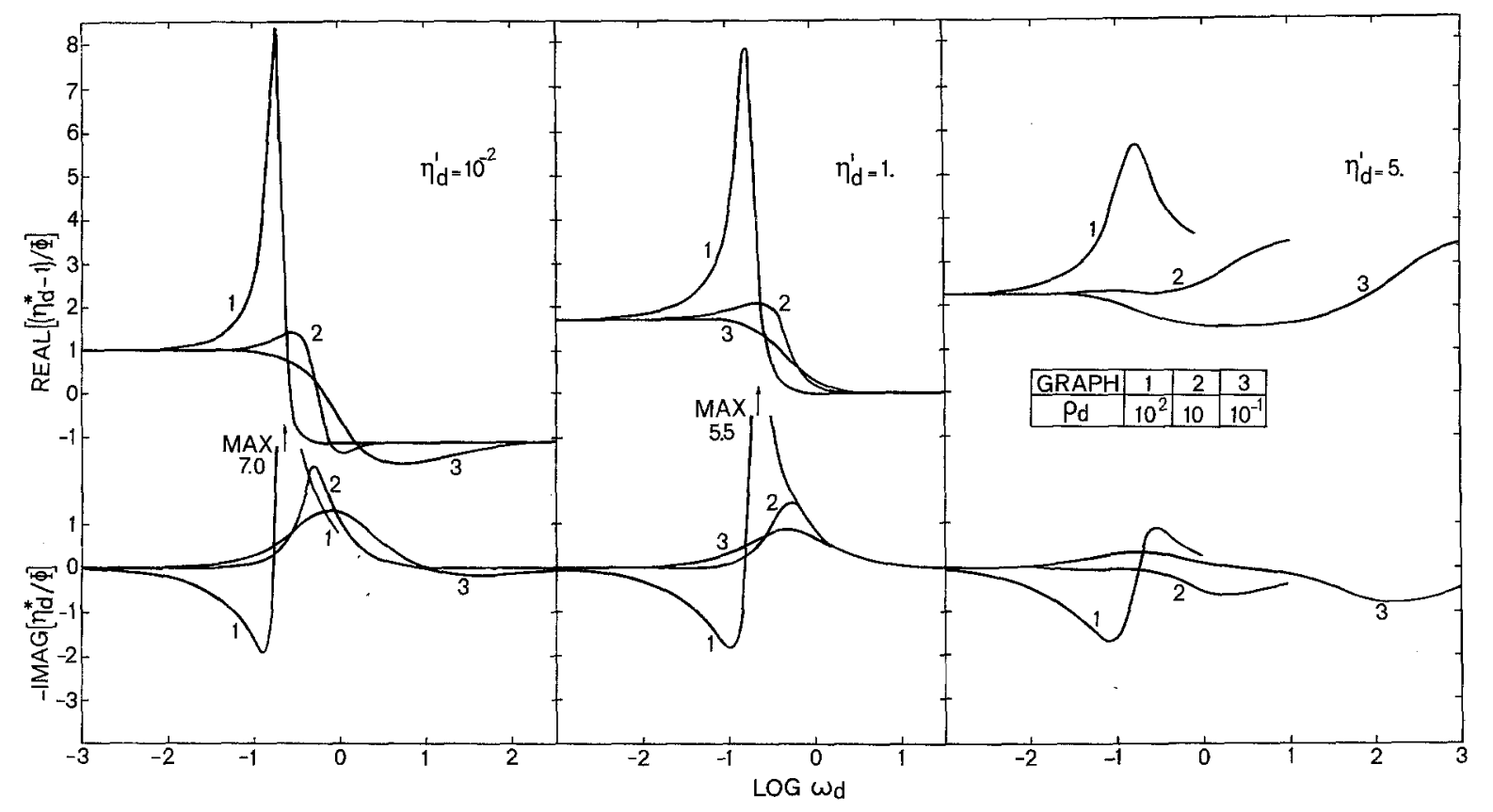

Fig. 4. The real and imaginary parts of $\left(\eta_{d}^{*}-1\right) / \Phi$ as a function of $\omega_{d}$ for such $\rho_{d}$ values that interfacial tension, shear and inertia forces are on the same order of magnitude

tude as the shear forces at the interface internalexternal phase.

In figure 3 interfacial tension forces are dominant for $\omega_{d} \ll \lambda_{d}^{-1}$ and shear forces are dominant for $\omega_{d} \gg \lambda_{d}^{-1}$ apart from inertia forces.
Introducing of inertia forces makes the picture more complicated. We can expect appreciable influence of inertia forces when the argument of the spherical Bessel and Hankel functions is on the order of unity or more, for example $\omega_{d} \rho_{d} \geq 1$. In order to demonstrate the 
effect of inertia on $\eta_{d}^{*}$ separately from the wellknown effects at $\omega_{d}=\lambda_{d}^{-1}$ we have chosen two $\rho_{d}$ values which satisfy the conditions $\rho_{d} \ll \lambda_{d}$ and $\rho_{d} \gg \lambda_{d}$.

For intermediate $\rho_{d}$ values inertia effects interfere with the normal viscoelastic effects. The qualitative character changes strongly with relatively small changes in the parameter values. For reasons of clarity such effects are not included in figure 3 , but they are shown separately in figure 4 . In figure 3 for $\rho_{d}=10^{3}$ only increasing and decreasing paths of the curves of the real and imaginary part of $\left(\eta_{d}^{*}-1\right) / \Phi$ can be calculated. For $\eta_{d}^{*}=10^{-2}$ the behaviour caused by inertia can be characterized as an increase of the real part of $\left(\eta_{d}^{*}-1\right) / \Phi$ with a point of inflection and an increase of the imaginary part of $\left(\eta_{d}^{*}-1\right) / \Phi$ with a maximum. In both cases the imaginary part is positive. The behaviour is strongly dependent on $\eta_{d}^{\prime}$. As expected, $\left(\eta_{d}^{*}-1\right) / \Phi=\left(\eta_{01}^{*}-1\right) / \Phi$ if $\eta_{d}^{\prime}=1$ and $\rho_{d} \ll \lambda_{d}$. In the limit for $\eta_{d}^{\prime} \rightarrow \infty$ our results approach those of the exact expression for solid spheres $\left.(17)^{*}\right)$ :

$\frac{\eta_{d}^{*}-1}{\Phi}=\frac{15+15 \ddot{i k}-6 k^{2}-i k^{3}}{6(1+i k)}$.

This function depends on $k^{2}=-i \omega_{d} \rho_{d}$. As an example it is plotted for $\rho_{d}=10^{-2}$ as a function of $\omega_{d}$ and compared with our result for $\eta_{d}^{\prime}=10^{4}$. The agreement is very satisfactory.

The complex behaviour of $\eta_{d}^{*}$ for intermediate values of $\rho_{d}$ between $10^{-2}$ and $10^{3}$ is shown in figure 4. The influence of inertia is intermediate that for low and high $\rho_{d}$ values. The influence of normal viscoelastic effects is easily recognized. The strong dependence of $\eta_{d}^{\prime}$ which is present in figure 3 is also present here. Compared with figure 3 the large minima of the imaginary part of $\eta_{d}^{*} / \Phi$ are unexpected. They can be regarded as a typical effect of the interference of the interfacial tension, shear and inertia forces when they are on the same order of magnitude.

The calculated changes in $\eta_{d}^{*}$ caused by inertia forces are not a common feature of linear viscoelasticity. The interpretation in terms of a mechanical model with springs and dashpots alone is impossible.

*) Thanks are due to Dr. J. Hinch, who provided us with the relevant part of his thesis after the presentation of the preliminary results of our work on Euromech Colloquium 104, Leuven 4-7 September 1978.
However, introduction of mass elements in such models can easily produce a positive value for the imaginary part of $\eta_{d}^{*}$ and an increase in the real part of $\eta_{d}^{*}$. An example with two dashpots and one mass element is given in figure 5. The mechanical behaviour of this model can be characterized by a dynamic "viscosity" $\eta_{0}^{*}(\omega)=\eta_{0}^{\prime}(\omega)-i \eta_{0}^{\prime \prime}(\omega)$. The real and imaginary parts as a function of $\omega$ demonstrate a similar path as the curves on the righthand side in figure 3 . For solid spheres the solution is more difficult to interpret. For example, the real part of $\eta_{d}^{*}$ in eq. [29] is proportional to $\sqrt{\omega}$ for $\omega \rightarrow \infty$. Interpretation

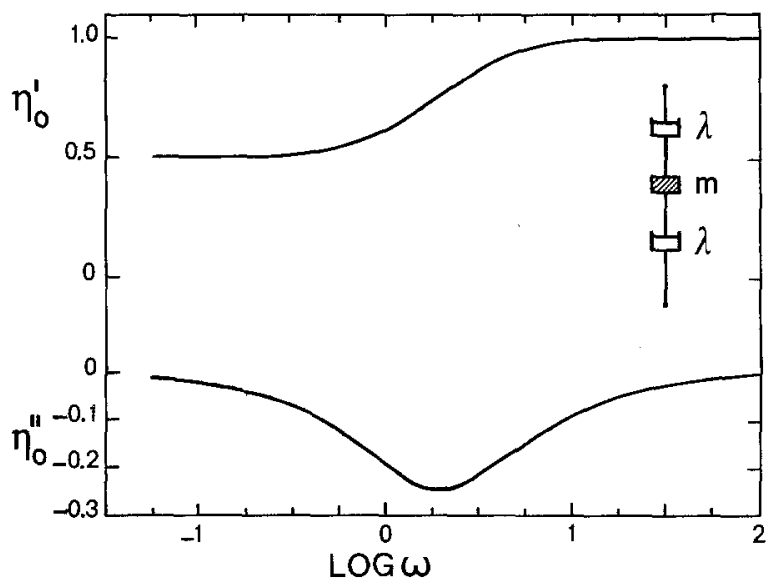

Fig. 5. Dynamical behaviour of the indicated massdashpot system for damping constant $\lambda=1$ and mass $m=1$

with a finite number of discrete mass, spring, or dashpot elements is impossible. It can be interpreted in terms of a mechanical model which involves a continuous element characterized by mass and dissipation per unit length. The real part of the impedance of such a mechanical transmission line is proportional to $\sqrt{\omega}$ for high frequencies.

We can conclude that the influence of inertia on $\eta_{d}^{*}$ in an oscillating pure straining motion can be appreciable. Strictly speaking, our results are only valid for oscillating pure straining motion, but they suggests, however, that inertia effects on $\eta_{d}^{*}$ of dispersions for oscillating shear flow as well are not always negligible.

$$
\begin{aligned}
& \text { Appendix } 1 \\
& \begin{aligned}
u= & \left\{A r^{-1} j_{2}(k r)+B r^{-1} j_{-3}(k r)+C r^{-4}+D r\right\} P_{2}, \\
v= & \frac{1}{6}\left\{A\left(3 r^{-1} j_{2}(k r)-k j_{3}(k r)\right)\right. \\
& +B\left(3 r^{-1} j_{-3}(k r)+k j_{-4}(k r)\right)-2 C r^{-4} \\
& +3 D r\} P_{2}^{\prime},
\end{aligned}
\end{aligned}
$$




$$
\begin{aligned}
& p=\eta\left\{-\frac{1}{3} C\right.\left.k^{2} r^{-3}+\frac{1}{2} D k^{2} r^{2}\right\} P_{2}+p_{0}, \\
& \hat{T}\langle r r\rangle=\eta\left\{2 A\left(r^{-2} j_{2}(k r)-k r^{-1} j_{3}(k r)\right)\right. \\
&+2 B\left(r^{-2} j_{-3}(k r)+k r^{-1} j_{-4}(k r)\right) \\
&+C\left(-8 r^{-5}+\frac{k^{2} r^{-3}}{3}\right) \\
&+\left.D\left(2-\frac{k^{2} r^{2}}{2}\right)\right\} P_{2}-p_{0}, \\
& \hat{T}\langle r \theta\rangle=\eta\left\{A\left(\left(r^{-2}-\frac{k^{2}}{6}\right) j_{2}(k r)+\frac{r^{-1} k}{3} j_{3}(k r)\right)\right. \\
&+B\left(\left(r^{-2}-\frac{k^{2}}{6}\right) j_{-3}(k r)-\frac{r^{-1} k}{3} j_{-4}(k r)\right) \\
&\left.+\frac{8}{3} C r^{-5}+D\right\} P_{2}^{\prime}, \\
& \hat{T}\langle\theta \theta\rangle=2 \eta\left\{( 1 - \frac { 3 } { 2 } \operatorname { c o s } ^ { 2 } \theta ) \left(A r^{-2} j_{2}(k r)\right.\right. \\
&+\left.B r^{-2} j_{-3}(k r)+D\right)-\frac{1}{2}\left(1-2 \cos ^{2} \theta\right) \\
&+\left(A r^{-1} k j_{3}(k r)-B k r^{-1} j_{-4}(k r)\right) \\
&\left.-\frac{1}{2}\left(3-7 \cos ^{2} \theta\right) C r^{-5}\right\}-p, \\
& \hat{T}\langle\phi \phi\rangle= 2 \eta\left\{A\left(-\frac{1}{2} r^{-2} j_{2}(k r)+\frac{1}{2} k r^{-1} j_{3}(k r) \cos ^{2} \theta\right)\right. \\
&+C\left(\frac{5}{2} \cos ^{2} \theta-\frac{1}{2}\right) r^{-5}+B\left(-\frac{1}{2} r^{-2} j_{-3}(k r)\right. \\
&\left.\left.-\frac{1}{2} k r^{-1} j_{-4}(k r) \cos ^{2} \theta\right)-\frac{1}{2} D\right\}-p,
\end{aligned}
$$

The functions $j_{2}, j_{3}, j_{-3}$, and $j_{-4}$ represent spherical Bessel functions, and $k^{2}=-i \omega \rho / \eta$.

\section{Acknowledgements}

Thanks are due to Dr. H. R. Eekhof for his contribution to the numerical calculations. We would also like to thank Prof. Dr. P. F. Mijnlieff for valuable discussions.

\section{Summary}

The dynamic viscosity of a dilute emulsion is calculated for a pure straining motion. The emulsion consists of almost spherical drops of a Newtonian fluid immersed in another Newtonian fluid. The oscillating velocity field of the flow is derived from the Navier-Stokes equation, in which the linear inertia term is included, whereas the non-linear one is neglected. The dynamic viscosity is determined with the aid of a cell model. The results are calculated numerically and typical results are presented graphically. The influence of inertia on the dynamic viscosity appears to be appreciable. Special cases presented in the literature, frequency or density zero and rigid spheres, are confirmed.

\section{Zusammenfassung}

Die dynamische Viskosität einer verdünnten Emulsion wird für eine reine Dehnströmung berechnet. Die Emulsion besteht aus nahezu sphärischen Tropfen einer newtonschen Flüssigkeit, die in einer anderen newtonschen Flüssigkeit verteilt sind. Das oszillierende Geschwindigkeitsfeld der Strömung wird mit Hilfe der Navier-Stokesschen Gleichung abgeleitet, wobei das lineare Trägheitsglied mitgenommen, die nicht-linearen Trägheitsglieder aber vernachlässigt werden. Die dynamische Viskosität wird mit Hilfe eines Zellenmodells ermittelt. Die Ergebnisse werden numerisch berechnet, und typische Ergebnisse werden graphisch dargestellt. Der Einfluß der Trägheit auf die dynamische Viskosität erweist sich als beträchtlich. Die Ergebnisse von in der Literatur schon behandelten Spezialfällen, bei denen die Frequenz oder die Dichte null gesetzt bzw. die Kugeln als starr angenommen worden waren, werden von uns bestätigt.

\section{References}

1) Oldroyd, J. G., Proc. Roy. Soc. A 218, 122 (1953).

2) Schowalter, W. R., C. E. Chaffey, H. Brenner, J. Coll. Interf. Sci. 26, 152 (1968).

3) Frankel, N. A., A. Acrivos, J. Fluid Mech. 44, 65 (1970)

4) Barthés-Biesel, D., A. Acrivos, Int. J. Multiphase Flow 1, 1 (1973). (1946)

5) Fröhlich, H., R. Sack, Proc. Roy. Soc. A 185, 415

6) Lamb, H., Hydrodynamics, Cambridge University Press (1975). (1972)

7) Hinch, J., Thesis Cambridge University, 1.21

Authors' address:

Ir. M. Oosterbroek, Ir. R. Tropper, Dr. J. Mellema

Department of Applied Physics

Twente University of Technology

Enschede (The Netherlands) 